\title{
Quality of Life in Children Post-Fundoplication for Gastroesophageal Reflux Disease
}

\author{
Shravya Pilli ${ }^{1}$, Ashish Jiwane ${ }^{1,2}$ and Usha Krishnan ${ }^{1,3 *}$ \\ ${ }^{1}$ School of Women's and Children's Health, University of New South Wales, Sydney, Australia \\ ${ }^{2}$ Department of Pediatric Surgery, Sydney Children's Hospital, Sydney, Australia \\ ${ }^{3}$ Department of Pediatric Gastroenterology, Sydney Children's Hospital, Sydney, Australia
}

Submission: November 07,2019; Published: November 18, 2019

*Corresponding author: Usha Krishnan, Department of Pediatric Gastroenterology, Sydney Children's Hospital, Sydney, Australia

\begin{abstract}
Objective: To explore quality of life (QOL) outcomes in children who have had a fundoplication surgery for the treatment of gastroesophageal reflux disease (GERD) using post-operative results of GERD-specific and pediatric-specific questionnaires.

Study Design: In this monocentric exploratory study, 115 patients who underwent fundoplication between 2006 and 2013 were contacted and provided with parent and child reports of three questionnaires - GERD-HRQL, PEDS-QL Gastrointestinal Symptoms Module and PEDS-QL Generic Core Scales. Data analysis on QOL outcomes were based on these responses.

Results: A total of 29 patients responded. The mean age was 11.26 years, $55.2 \%$ were male and $20.7 \%$ were neurologically impaired (NI). Neurologically normal children and children who had a concurrent gastrostomy insertion have significantly better QOL outcomes than their counterparts. Significant positive correlation was found between symptom based QOL and general QOL. While there was trend towards QOL being better in the medium-term group compared to the short-term group and in accordance to child perception compared to parental perception of QOL, statistical significance was not found.

Conclusion: Reflux-related QOL post-fundoplication has multiple influential factors. Developing research models which will permit assessment of QOL by comparing not only pre-surgical and post-surgical patients, but also QOL in children who are being treated with only anti-reflux medications and lifestyle modification at baseline and on therapy in both the short and long- term, are needed before the adoption of pediatric fundoplication surgeries for GERD can be justified.
\end{abstract}

Keywords: Quality of Life Fundoplication; Children; Hospitalizations; Gastroesophageal Reflux; Morbidity; Reflux symptoms; Fundoplication surgery; Laparoscopic; Pediatric gastroenterology; Neurological impairment

\section{Introduction}

When gastroesophageal reflux disease (GERD) is chronic, severe and resistant to medical therapy, it necessitates surgical intervention- fundoplication. It is the third 4 most common abdominal procedure performed by pediatric surgeons in the United States [1]. Children with comorbidity, primarily neurological impairment (NI), are often at a higher risk of severe GERD and more likely to need fundoplication. In fact, NI children account for nearly $40 \%$ of fundoplication's performed [2,3]. While there is documented evidence of the procedure's effectiveness in reducing reflux events, symptom recurrence, hospitalizations as well as its associated morbidity and complication rates [419], there has been minimal research assessing the relationship between reflux symptoms post-surgery and the child and family's QOL, an increasingly important patient care outcome.

\section{Objectives}

The purpose of this study was to explore post-operative QOL outcomes and reflux symptoms for up to 10 years in children who have had a fundoplication surgery for the treatment of GERD, using primarily pediatric-specific instruments. We aimed:

i. To determine mean scores of QOL and identify whether there was difference in QOL outcomes in children post fundoplication for GERD in the short-term ( $<5 \mathrm{yrs}$ ) compared to the medium-term (5-10yrs)

ii. To determine if number of reflux symptoms correlates with QOL

iii. To identify if there was a relationship between reflux symptom based QOL and general QOL 
iv. To identify if there was a difference in child and parental perception of the child's QOL

v. To identify if NI and concurrent gastrostomy with fundoplication influenced their current QOL status

\section{Methods}

\section{Recruitment}

This was a monocentric exploratory study. Participants were recruited from a database of children who had a fundoplication surgery between 2006 and 2013 at Sydney Children's Hospital (SCH). All participants were aged between 0-18 years at the time of surgery. Any patients that were lost to follow up were excluded. Parents of all patients were contacted:

a) During their regular follow up clinic visits, or

b) By mail, followed up with a phone call within 2-4 weeks to determine their decision.

In the age group under 5 years, only the parent completed the questionnaire. In older age groups, children were also requested to complete a child report if they were intellectually capable of doing so.

Results

\section{Data collection}

Three standardized questionnaires were provided with both child and parent reports:
a. GERD-HRQL: A GERD-specific questionnaire
b. PEDS-QL Gastrointestinal Symptoms Module: A pediatric-specific gastrointestinal questionnaire
c. PEDS-QL Generic Core Scales: A pediatric-specific general QOL questionnaire

\section{Statistical analysis}

Statistical analysis of results was performed using SPSS Statistics 23. Descriptive statistics, wilcoxon signed rank test, mann-whitney U test, spearman's correlation and kruskall wallis $\mathrm{H}$ test were used. Approval for this study was obtained from the Human Research Ethics Committee at SCH, Randwick (LNR/15/ SCHN/451).

Data supporting results reported in this article has been stored in a secure facility at Department of Pediatric Gastroenterology, Sydney Children's Hospital, Sydney, Australia. There was no funding involved for this clinical study.

\section{Patient responses}

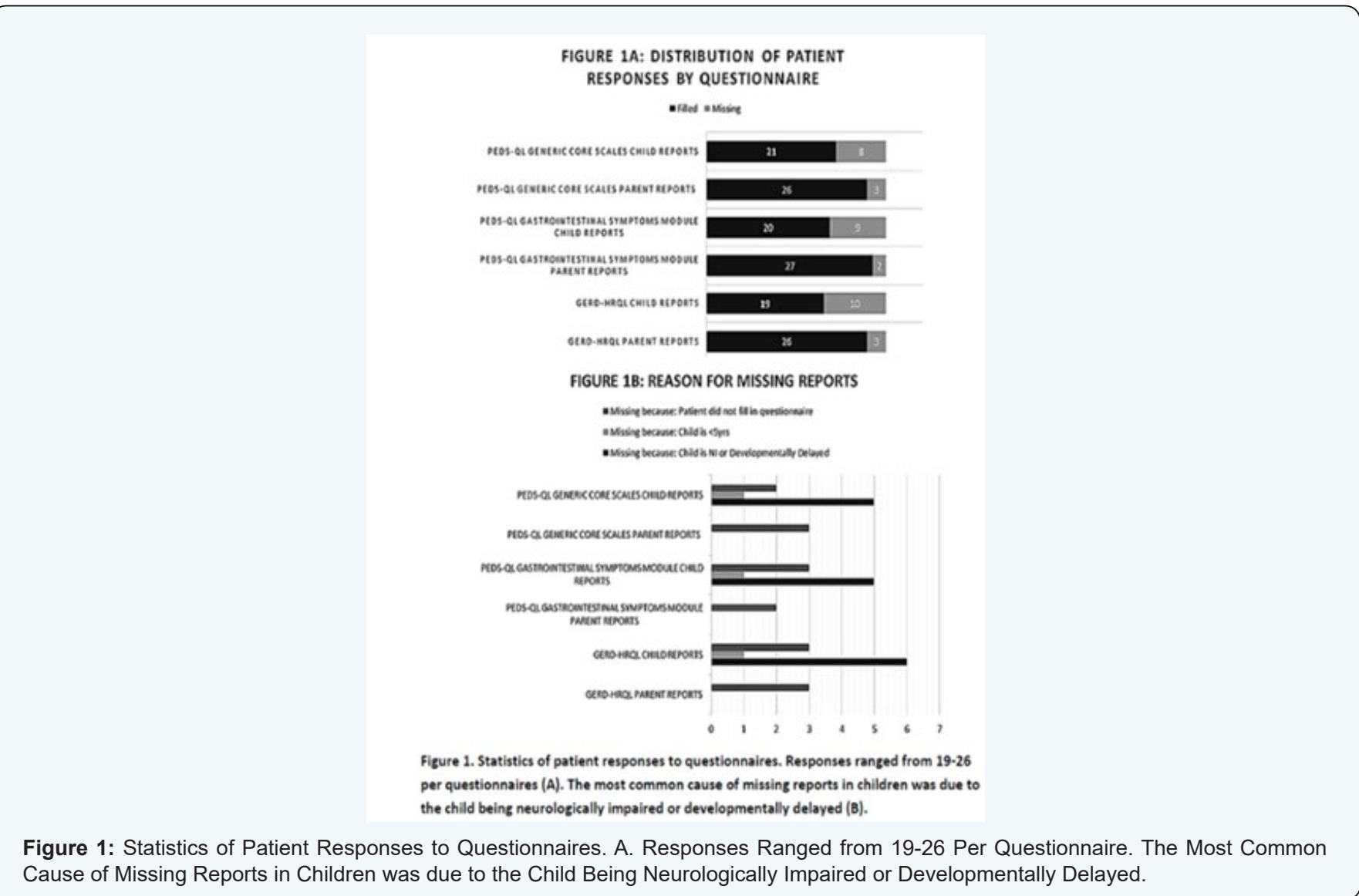

The 115 patients who had a fundoplication between 2006 (Figure 1a). Figure 1b depicts reports identified as missing with and 2013 were contacted. A total of 29 responses were received reasons: 
i. The child was unable to fill in the questionnaire as a result of being $\mathrm{NI}$

ii. The child was unable to fill in the questionnaire being under 5 years of age

iii. The parent or child chose not to fill in the questionnaire

\section{Patient demographics}

The mean age of participants at the time of administration of questionnaires was 135.1 months (SD 54.24 months), including 16 males (55.2\%) and 13 females (44.8\%). 6 patients had NI (20.7\%).

\section{Details of fundoplication and surgical outcomes}

All patients had a Nissen fundoplication (27 Laparoscopic,
2 Open). At the time of surgery, mean age was 62.3 months, SD 56.96 months. 1 patient had a re-do operation. 10 patients (34.5\%) had a concurrent gastrostomy insertion. 16 patients (55.2\%) had recorded GERD investigations performed presurgery, of which $13(81.3 \%)$ indicated abnormal results. 8 patients (27.6\%) had recorded GERD investigations performed post-surgery for presumed recurrence of GERD symptoms which only $1(12.5 \%)$ indicated an abnormal result. 15 patients (51.7\%) have been restarted on anti-reflux medications postfundoplication. 2 patients are dependent on gastrostomy feeds, 1 patient combines both oral and gastrostomy feeds and the remaining 26 patients all feed orally. 13 patients (44.8\%) were hospitalised in the last year, of which 9 patients $(31.0 \%)$ were admitted due to a gastrointestinal complaint. A summary of patient characteristics is outlined in Table 1.

Table 1: Characteristics of Patients in Study Group.

\begin{tabular}{|c|c|}
\hline Patient Characteristic & Statistic \\
\hline Mean age at fundoplication, mo. (SD) & $62.3(56.96)$ \\
\hline Mean age at study questionnaire, mo. (SD) & $135.1(54.24)$ \\
\hline \multicolumn{2}{|l|}{ Gender, n (\%) } \\
\hline Male & $16(55.2 \%)$ \\
\hline Female & $13(44.8 \%)$ \\
\hline \multicolumn{2}{|l|}{ Time period between surgery and QOL assessment } \\
\hline Short-term $(<5$ yrs. $)$ & $11(37.9 \%)$ \\
\hline Medium-term (5-10 yrs.) & $18(62.1 \%)$ \\
\hline \multicolumn{2}{|l|}{ Comorbidity, n (\%) } \\
\hline NI & $6(20.7 \%)$ \\
\hline TOF Repair & $2(6.9 \%)$ \\
\hline \multicolumn{2}{|l|}{ Fundoplication, n (\%) } \\
\hline Nissen & $29(100 \%)$ \\
\hline Laparoscopic & $27(93.1 \%)$ \\
\hline Open & $2(6.9 \%)$ \\
\hline Re-do operation & $1(3.4 \%)$ \\
\hline Concurrent insertion of gastrostomy & $10(34.5 \%)$ \\
\hline \multicolumn{2}{|l|}{ Investigations for GERD Pre-Surgery, n } \\
\hline Recorded & 16 \\
\hline Abnormal (\% of Recorded) & $13(81.3 \%)$ \\
\hline \multicolumn{2}{|l|}{ Investigations for GERD Post-Surgery, n } \\
\hline Recorded & 8 \\
\hline Abnormal (\% of Recorded) & $1(12.5 \%)$ \\
\hline Patients restarted on anti-reflux medications & $15(51.7 \%)$ \\
\hline \multicolumn{2}{|l|}{ Type of Feeding, n (\%) } \\
\hline Oral & $26(89.7 \%)$ \\
\hline Oral + PEG & $1(3.4 \%)$ \\
\hline PEG & $2(6.9 \%)$ \\
\hline \multicolumn{2}{|l|}{ Hospitalisations in the last year, $n(\%)$} \\
\hline Patients hospitalised & $13(44.8 \%)$ \\
\hline Patients hospitalised due to GI complaint & $9(31.0 \%)$ \\
\hline
\end{tabular}




\section{QOL outcomes}

The PEDS-QL gastrointestinal symptoms module and PEDS-QL generic core scales both produces scores which are

Table 2a: Mean QOL Scores by Questionnaire.

\begin{tabular}{|c|c|c|c|c|c|}
\hline \multirow{2}{*}{ Questionnaire } & \multicolumn{2}{|c|}{ Parent Report } & \multicolumn{2}{c|}{ Child Report } & \multicolumn{2}{c|}{ Mean } \\
\cline { 2 - 6 } & $\begin{array}{c}\text { Response } \\
\text { Number }\end{array}$ & n (SD)* & $\begin{array}{c}\text { Response } \\
\text { Number }\end{array}$ & n (SD)* & Difference n \\
\hline PEDS-QL Gastrointestinal Symptoms Module Total Score & 27 & $74.29(14.78)$ & 20 & $75.66(14.79)$ & 1.37 \\
\hline PEDS-QL Gastrointestinal Symptoms Module Symptom Score & 27 & $73.67(15.38)$ & 20 & $74.98(14.90)$ & 1.31 \\
\hline GERD-HRQL Total Score & 26 & $12.54(11.95)$ & 21 & $9.76(12.68)$ & -2.78 \\
\hline GERD-HRQL Symptom Score & 26 & $11.58(11.18)$ & 21 & $8.86(11.54)$ & -2.72 \\
\hline PEDS-QL Generic Core Scales Total Score & 26 & $68.52(24.54)$ & 20 & $76.09(18.79)$ & 7.57 \\
\hline PEDS-QL Generic Core Scales Psychosocial Summary Score & 26 & $67.63(23.83)$ & 20 & $74.42(20.29)$ & 6.79 \\
\hline PEDS-QL Generic Core Scales Physical Summary Score & 26 & $69.83(29.71)$ & 20 & $79.22(19.06)$ & 9.39 \\
\hline
\end{tabular}

*All Numbers Were Rounded to the Nearest Hundredth of a Decimal Point.

Table 2b: QOL Scores in the Short-Term Vs Medium-Term.

\begin{tabular}{|c|c|c|c|c|c|c|c|c|}
\hline \multirow{3}{*}{ Medium-Term (5-10 yrs.) } & \multicolumn{4}{|c|}{ Parent Report } & \multicolumn{4}{|c|}{ Child Report } \\
\hline & \multicolumn{2}{|c|}{ Short-Term ( $<5$ yrs.) } & \multicolumn{2}{|c|}{$\begin{array}{l}\text { Medium-Term (5-10 } \\
\text { yrs.) }\end{array}$} & \multicolumn{2}{|c|}{$\begin{array}{c}\text { Short-Term }(<5 \\
\text { yrs.) }\end{array}$} & \multicolumn{2}{|c|}{$\begin{array}{c}\text { Medium-Term (5-10 } \\
\text { yrs.) }\end{array}$} \\
\hline & $\begin{array}{l}\text { Response } \\
\text { Number }\end{array}$ & $\mathbf{n}^{*}$ & $\begin{array}{l}\text { Response } \\
\text { Number }\end{array}$ & $\mathbf{n}^{*}$ & $\begin{array}{l}\text { Response } \\
\text { Number }\end{array}$ & $\mathbf{n}^{*}$ & $\begin{array}{l}\text { Response } \\
\text { Number }\end{array}$ & $\mathbf{n}^{*}$ \\
\hline $\begin{array}{l}\text { PEDS-QL Gastrointestinal Symptoms Module } \\
\text { Total Score }\end{array}$ & 10 & 69.75 & 17 & 76.96 & 8 & 71.92 & 12 & 78.15 \\
\hline $\begin{array}{l}\text { PEDS-QL Gastrointestinal Symptoms Module } \\
\text { Symptom Score }\end{array}$ & 10 & 68.38 & 17 & 76.79 & 8 & 70.96 & 12 & 77.66 \\
\hline GERD-HRQL Total Score & 10 & 15.1 & 16 & 10.94 & 8 & 9.88 & 13 & 9.69 \\
\hline GERD-HRQL Symptom Score & 10 & 14.6 & 16 & 9.69 & 8 & 9.13 & 13 & 8.69 \\
\hline PEDS-QL Generic Core Scales Total Score & 10 & 72.72 & 16 & 65.9 & 8 & 75.41 & 12 & 76.54 \\
\hline $\begin{array}{l}\text { PEDS-QL Generic Core Scales Psychosocial } \\
\text { Summary Score }\end{array}$ & 10 & 73.33 & 16 & 64.06 & 8 & 74.79 & 12 & 74.17 \\
\hline $\begin{array}{l}\text { PEDS-QL Generic Core Scales Physical } \\
\text { Summary Score }\end{array}$ & 10 & 71.56 & 16 & 68.75 & 8 & 76.56 & 12 & 80.99 \\
\hline
\end{tabular}

*All Numbers were Rounded to the Nearest Hundredth of a Decimal Point.

The mean QOL scores by questionnaire are summarized in Table 2a. The use of the PEDS-QL gastrointestinal symptoms module and GERD-HRQL enabled us to view QOL in the context of reflux symptoms. Including the PEDS-QL generic core scales, enabled us to compare with the child's general QOL. The QOL scores in all three questionnaires indicated better outcomes in the child reports compared to the parent reports. $62.1 \%$ of patients had their QOL outcomes measured in the medium-term. In both parent and child reports, there was a trend towards the QOL scores being better in the medium-term compared to the short-term group, as shown in Table $2 \mathrm{~b}$. However, none of these differences were statistically significant $(\mathrm{p}<0.05)$.

The child reports of the PEDS-QL Gastrointestinal Module were used to determine if the number of reflux symptoms in the children was associated with their QOL. Symptoms that were considered included stomach pain, stomach discomfort when eating, food and drink limits, trouble swallowing, heartburn, transformed to a 0-100 scale. The height of the score positively correlates to the QOL. The GERD-HRQL has a score scale ranging from $0-75$, with the height of the score negatively correlating to the QOL. 
neurologically normal children, children who had a concurrent gastrostomy insertion and those who are currently not on any anti-reflux medications had either significantly better general or symptom related QOL scores than their counterparts, accounting for a better QOL.
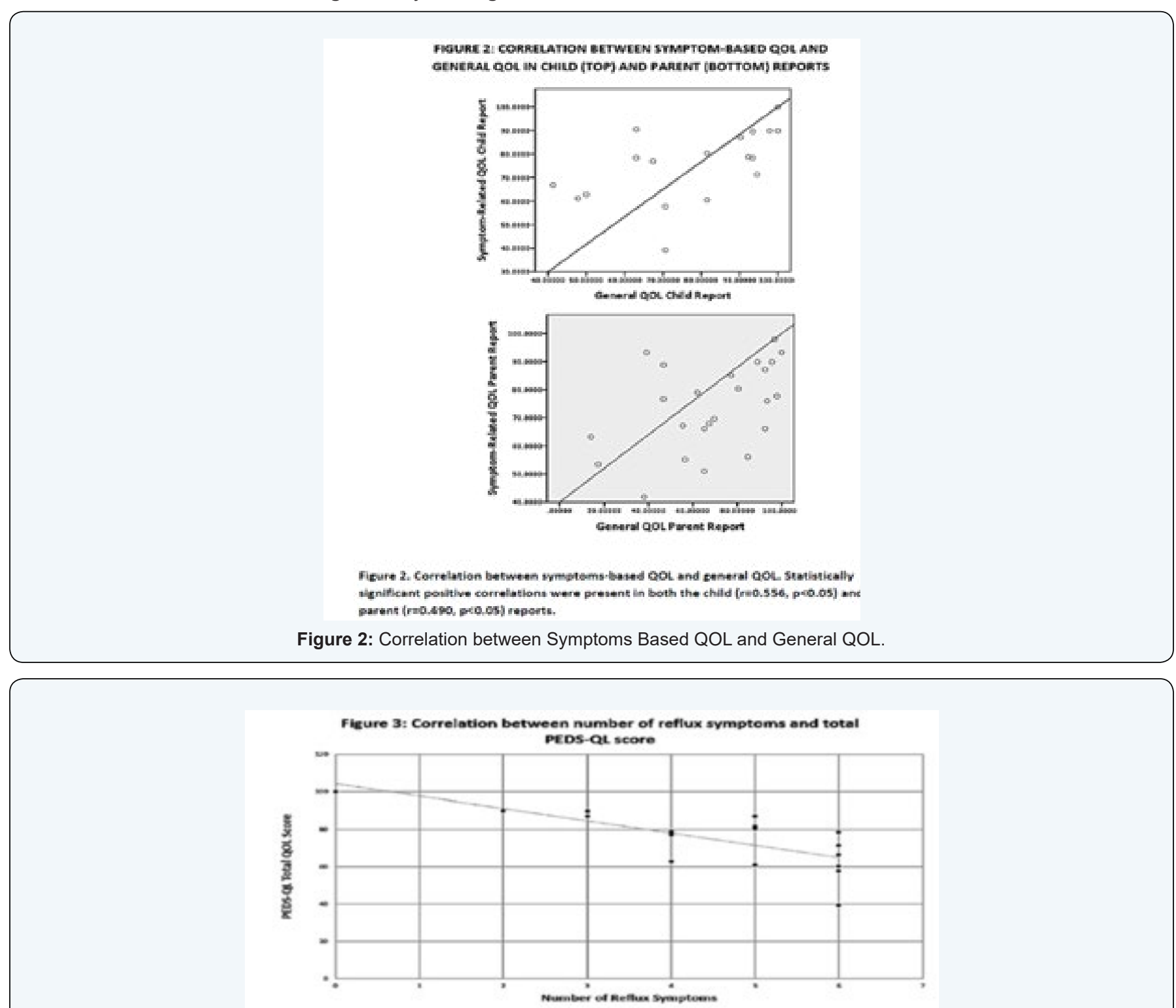

Figure 3. Correlation between number of reflux symptoms and total PEDS-QL score. The fewer the number of symptoms, the better the QOL scores of the children (r=0.729. peo.es).

Figure 3: Correlation between Number of Reflux Symptoms and Total PEDS-QL Score.

\section{Discussion}

The importance of clinically assessing children more holistically is becoming increasingly apparent, with an increased focus on integrating patient-centred outcomes in the consideration of new therapies, reinforcing the shift in medicine to beyond just the physical symptoms [20]. With chronic conditions like GERD, it is vital to determine fundoplication improved not only reflux symptoms but also QOL post surgery in children. It is also improtant to delineate if reflux symptoms showed any form of correlation to QOL post-fundoplication.

\section{Child and parental perception}

In the past, QOL studies looking at fundoplication [21-24], relied primarily on parental perception without taking into account the child's views of the impact of the illness and the efficacy of the treatment [25]. This study is one of the first to look at both parent and child reports. We found a difference, 
albeit non-significant, when comparing parent and child reports of all three questionnaires, with a trend towards children consistently reporting a better QOL than parents. Eiser \& Morse [26], in their review of QOL measures in children with chronic disease, support this finding in their suggestion that parental expectations and hopes for their child, comparison of their child with the development of others, additional life stresses and their own mental health can serve as negative influential factors [27].

It is well-known that there are different aspects that influence the QOL of a child compared to that of an adult [28]. This creates issues in the overlap of the use of instruments across the two groups. While Engelmann et al. used a modified version of the GIQLI, it was still based off an adult questionnaire. Both O'Neill \& Pacilli et al. $[22,23]$ used the same untitled non-standardised questionnaires, which raises uncertainty about the reliability of the paediatric focus. In the present study, we adopted two paediatric-specific PEDS-QL questionnaires. However, with the lack of a GERD-specific paediatric instrument, we resorted to the use of the adult-based GERD-11.

HRQL questionnaire. Subsections of the PEDS-QL also relate to GERD specific symptoms. Interestingly the mean scores of 76.09 in our population post-fundoplication was similar to the pre-fundoplication scores in Mauritz et al study [29] which also adopted the PEDS-QL generic core scales as a measurement of health-related QOL for children that underwent laparoscopic fundoplication for GERD, although we did not have prefundoplication scores to compare with.

\section{Short-term and medium-term QOL outcomes}

While the majority of previous paediatric QOL studies restricted their measurement of QOL to a very short-term of usually up to 6 months [21-24], this is the only study which has looked at children up to 10 years post-fundoplication. Pacilli et al. [23] who performed a follow-up assessment at 4 years found that there was a progressive improvement in QOL in all domains from the pre-surgical status, to 6 months and later at 4 years. This matches our results, which generally showed a better QOL in children in the medium-term group compared to the shortterm group.

\section{Number of reflux symptoms and QOL}

While we were unable to compare pre-and postfundoplication scores of these patients to determine if there was an improvement in QOL, we were able to identify that in those children with fewer reflux symptoms post-operatively, better QOL score were reported. This highlights the importance of reflux symptoms in determining the QOL of the child. It also highlights the role of fundoplication in improving QOL by relieving GERD symptoms.

\section{Symptom-based and general QOL}

In the past, studies have adopted single instruments [21-24] that combined both symptoms based and general QOL domains into the same questionnaire. While this has the benefit of a more likely higher response rate with the reduced number of forms to be filled in by patients, we are the first study to have adopted the use of separate distinct questionnaires to identify whether in fact there was a relationship between symptomatic change postfundoplication and general QOL, including physical, emotional, behavioural and school functioning. We found a significant positive correlation $(\mathrm{p}<0.05)$ between the two, suggesting that with an improvement of symptoms, children are more likely to function better psychosocially.

\section{QOL of NI children}

NI children, often with multiple co-morbidities and a reduced level of independence are prone to a poorer QOL than neurologically normal children. They are reported to experience more severe GERD and hence NI children form a large proportion of children that undergo fundoplication. In our study, NI children had a poorer QOL compared to neurologically normal children post-fundoplication $(\mathrm{p}<0.05)$, which is similar to the results of Pacilli \& Engelmann et al, [21,23]. Despite an identified improvement in QOL post-fundoplication in NI children, their rates of fundoplication failure and complications are higher than neurologically normal children [30]. This corresponds to the gradual decrease in the proportion of NI 13 children undergoing fundoplication in the United States in spite of the greater severity of disease [3].

\section{Concurrent gastrostomy with fundoplication}

The concurrent insertion of agastrostomy with fundoplication has been suggested to be associated with a better QOL. The venting of the gastrostomy tube decompresses the stomach and is believed to ease the discomfort caused by gas-bloat which has been reported post-fundoplication. It is also believed to be associated with an increased ease of feeding compared to pre-surgical nasogastric tubes [23]. This is especially so in NI children with an unsafe swallow and aspiration risk. However, while past studies have shown no significant improvement in the QOL of children with a concurrent gastrostomy when compared to those without one post-fundoplication, our present study, where $34.5 \%$ of children had a concurrent gastrostomy, showed a significantly better GERD-specific QOL in children with a concurrent gastrostomy $(\mathrm{p}<0.01)$.

The main limitation in our study was its small sample size resulting in low power for obtaining statistical significance and strength in correlation. While the majority of patients did show an interest, their personal time constraints limited our response rate. Ideally, the testing of pre-surgical QOL outcomes would have provided a baseline comparison to determine the 'impact' of fundoplication in this study population. However, the present study was constrained in this assessment having focused on a retrospective database with could have potentially resulted in a recall bias.

b , particularly in the medium-term group. While this creates potential for skewed results, this study has created scope in raising questions about the assessment of reflux symptoms 
in relation to QOL that may have previously been unidentified. Despite its limitations, it was the first Australian study to look at post-fundoplication QOL outcomes and provide valuable insight into factors that were overlooked in previous studies. This is one of the first studies to adopt paediatric-specific QOL instruments and to look at both child and parental perception of the child's QOL. It highlights the issues associated with parent-proxy reporting of their child's health and the need for a more direct child involvement. It is also the first time QOL was observed beyond just 4 years post-fundoplication, highlighting the importance of long-term follow-up of these patients. The study was able to provide evidence to support the claims made by literature of the associated benefit of a concurrent gastrostomy with fundoplication. Expanding the assessment of refluxrelated QOL outcomes beyond just pre- and post-fundoplication patients to the assessment of children with GERD that are being treated solely by anti-reflux medications and solely by lifestyle modification will assist in determining whether the current high trend in paediatric fundoplications being performed is valid. With improvements in this relatively new and continually evolving field of QOL assessment, it will be possible to work towards identifying the sub-populations most of children benefitting from fundoplication surgery and identifying patientcentred outcomes to further improve the post-surgical QOL of these children.

\section{References}

1. Fox D, Morrato E, Campagna EJ, Rees DI, Dickinson LM, et al. (2011) Outcomes of Laparoscopic Versus Open Fundoplication in Children's Hospitals: 2005-2008. Pediatrics 127(5): 872-880.

2. Goldin AB, Garrison M, Christakis D (2009) Variations between hospitals in antireflux procedures in children. Archives of Pediatrics \& Adolescent Medicine 163(7): 658-663.

3. Lasser MS, Liao JG, Burd RS (2006) National trends in the use of antireflux procedures for children. Pediatrics 118(5): 1828-1835.

4. Allal H, Captier G, Lopez M, Forgues D, Galifer RB (2001) Evaluation of 142 consecutives laparoscopic fundoplications in children: Effects of the learning curve and technical choice. J Pediatr Surg 36(6): 921-926.

5. Pascoe E, Falvey T, Jiwane A, Henry G, Krishnan U (2016) Outcomes of fundoplication for pediatric gastroesophageal reflux disease. Pediatr Surg Int 32(4): 353-361.

6. Bourne MC, Wheeldon C, MacKinlay GA, Munro FD (2003) Laparoscopic Nissen fundoplication in children: 2-5-year follow-up. Pediatric Surgery International 19(7): 537-539.

7. Dedinsky GK, Vane DW, Black CT, Turner MK, West KW, et al. (1987) Complications and reoperation after Nissen fundoplication in childhood. Am J Surg 153(2): 177-183.

8. Esposito C, Montupet P, Amici G, Desruelle P (2014) Complications of laparoscopic antireflux surgery in childhood. Surg Endosc 14(7): 622624.

9. Esposito C, Montupet P, Zee D, Settimi A, Paye-Jaouen A, et al. (2006) Long-term outcome of laparoscopic Nissen, Toupet, and Thal antireflux procedures for neurologically normal children with gastroesophageal reflux disease. Surg Endosc 20(6): 855-858.
10. Fonkalsrud EW, Ashcraft KW, Coran AG, Ellis DG, Grosfeld JL, et al (1998) Surgical treatment of gastroesophageal reflux in children: a combined hospital study of 7467 patients. Pediatrics 101(3 Pt 1): 419422.

11. Fonkalsrud EW, Bustorff-Silva J, Perez CA, Quintero R, Martin L, et al. (1999) Antireflux surgery in children under 3 months of age. J Pediatr Surg 34(4): 527-531.

12. Gilger MA, Yeh C, Chiang J, Dietrich C, Brandt ML, et al. () Outcomes of surgical fundoplication in children. Clin Gastroenterol Hepatol 2(11): 978-984.

13. Kubiak R, Spitz L, Kiely EM, Drake D, Pierro A (1999) Effectiveness of fundoplication in early infancy. Journal of Pediatr Surg 34(2): 295-299.

14. Leung L, Wong CWY, Chung PHY, Wong KKY, Tam PKH (2014) Laparoscopic Nissen fundoplication for gastro-oesophageal reflux disease in infants. Pediatr Surg Int 31(1): 83-88.

15. Mathei J, Coosemans W, Nafteux P, Decker G, Leyn P, et al. (2007) Laparoscopic Nissen fundoplication in infants and children: analysis of 106 consecutive patients with special emphasis in neurologically impaired vs. neurologically normal patients. Surg Endosc 22(4): 1054-1059.

16. Meehan JJ, Georgeson KE (2014) Laparoscopic fundoplication in infants and children. Surg Endosc 10(12): 1154-1157.

17. Rothenberg SS (2013) Two decades of experience with laparoscopic nissen fundoplication in infants and children: a critical evaluation of indications, technique, and results. J Laparoendosc Adv Surg Tech A 23(9): 791-794.

18. Shariff F, Kiely E, Curry J, Drake D, Pierro A, et al. (2010) Outcome after laparoscopic fundoplication in children under 1 year. J Laparoendosc Adv Surg Tech A 20(7): 661-664.

19. Spillane AJ, Currie B, Shi E (1996) Fundoplication in Children: Experience with 106 Cases. A Aust N Z J Surg 66(11): 753-756.

20. Reeve BB, Wyrwich KW, Wu AW, Velikova G, Terwee CB, et al. (2013) ISOQOL recommends minimum standards for patient-reported outcome measures used in patient-centered outcomes and comparative effectiveness research. Qual Life Res 22(8): 1889-1905.

21. Engelmann C, Gritsa S, Ure BM (2010) Impact of laparoscopic anterior 270 degrees fundoplication on the quality of life and symptoms profile of neurodevelopmentally delayed versus neurologically unimpaired children and their parents. Surgi Endosc 24(6): 1287-1295.

22. O’Neill JK, O'Neill PJ, Goth-Owens T, Horn B, Cobb LM (1996) Care-giver evaluation of anti-gastroesophageal reflux procedures in neurologically impaired children: what is the real-life outcome? J Pediatr Surg 31(3): 375-380.

23. Pacilli M, Eaton S, McHoney M, Kiely EM, Drake DP, et al. (2014) Fouryear follow-up of a randomized controlled trial comparing open and laparoscopic Nissen fundoplication in children. Archives of disease in childhood 99(6): 516-521.

24. Srivastava R, Downey EC, Feola P, Samore M, Coburn L, et al. (2007) Quality of life of children with neurological impairment who receive a fundoplication for gastroesophageal reflux disease. J Hosp Med 2(3): 165-173.

25. Petrie KJ, Weinman J (1997) Perceptions of health and illness: Current research and applications: Taylor \& Francis.

26. Eiser C, Morse R (2001) A review of measures of quality of life for children with chronic illness. Archives of disease in childhood. 84(3): 205211.

27. Eiser C, Jenney ME (1996) Measuring symptomatic benefit and quality of life in pediatric oncology. British journal of cancer. 73(11): 13131316. 
28. Eiser C, Morse R (2001) Quality-of-life measures in chronic diseases of childhood: Core Research. Health Technol Assess 5(4): 151-157.

29. (2015) $14^{\text {th }}$ World Congress of Endoscopic Surgery and $22^{\text {nd }}$ International Congress of the European Association for Endoscopic Surgery (EAES) Paris, France, 25-28 June 2014. Surgical endoscopy 29(1): 1-74.

This work is licensed under Creative Commons Attribution 4.0 License

DOI: 10.19080/ARGH.2019.14.555882
30. Vandenplas Y, Rudolph CD, Di Lorenzo C, Hassall E, Liptak G, et al. (2009) Pediatric gastroesophageal reflux clinical practice guidelines: joint recommendations of the North American Society for Pediatric Gastroenterology, Hepatology, and Nutrition (NASPGHAN) and the European Society for Pediatric Gastroenterology, Hepatology, and Nutrition (ESPGHAN). J Pediatr Gastroenterol Nutr 49(4): 498-547.

\section{Your next submission with JuniperPublishers will reach you the below assets}

- Quality Editorial service

- Swift Peer Review

- Reprints availability

- E-prints Service

- Manuscript Podcast for convenient understanding

- Global attainment for your research

- Manuscript accessibility in different formats

( Pdf, E-pub, Full Text, audio)

- Unceasing customer service

Track the below URL for one-step submission https://juniperpublishers.com/online-submission.php 